\title{
CCL: A Portable and Tunable Collective Communication Library for Scalable Parallel Computers
}

\author{
Vasanth Bala, Jehoshua Bruck, Senior Member, IEEE, Robert Cypher, Member, IEEE, Pablo Elustondo, Alex Ho, \\ Ching-Tien Ho, Member, IEEE, Shlomo Kipnis, Member, IEEE, and Marc Snir, Senior Member, IEEE
}

\begin{abstract}
A collective communication library for parallel computers includes frequently used operations such as broadcast, reduce, scatter, gather, concatenate, synchronize, and shift. Such a library provides users with a convenient programming interface, efficient communication operations, and the advantage of portability. A library of this nature, the Collective Communication Library (CCL), intended for the line of scalable parallel computer products by IBM, has been designed. CCL is part of the parallel application programming interface of the recently announced IBM 9076 Scalable POWERparallel System 1 (SP1). In this paper, we examine several issues related to the functionality, correctness, and performance of a portable collective communication library while focusing on three novel aspects in the design and implementation of CCL: 1) the introduction of process groups, 2) the definition of semantics that ensures correctness, and 3) the design of new and tunable algorithms based on a realistic point-to-point communication model.
\end{abstract}

Index Terms - Collective communication algorithms, collective communication semantics, message-passing parallel systems, portable library, process group, tunable algorithms.

\section{INTRODUCTION}

$\mathbf{T}$ THE need for collective communication arises frequently in parallel computation. Collective communication operations simplify the programming of applications for parallel computers, facilitate the implementation of efficient communication schemes on various machines, promote the portability of applications across different architectures, and reflect conceptual grouping of processes. In particular, collective communication is extensively used in many scientific applications

\footnotetext{
Manuscript received June 25, 1993; revised November 10, 1993. This work was done while V. Bala and S. Kipnis were with IBM T. J. Watson Research Center, and while J. Bruck, R. Cypher, and P. Elustondo were with IBM Almaden Research Center.

V. Bala is with the HP Laboratory, Cambridge, MA USA (e-mail: was@hpl.hp.com)

J. Bruck is with the California Institute of Technology, Mail Code 116-81, Pasadena, CA 91125 USA (e-mail: bruck@ systems.caltech.edu).

A. Ho and C.-T. Ho are with the IBM Research Division, Almaden Research Center, San Jose, CA 95120-6099 USA (e-mail: \{alexho,ho\}@almaden.ibm.com).

R. Cypher is with the Computer Science Department, the Johns Hopkins University, Baltimore, MD 21218 USA (e-mail: cypher@cs.jhu.edu).

M. Snir is with IBM Research Division, T.J. Watson Research Center, Yorktown Heights, NY 10598 USA (e-mail: snir@watson.ibm.con).

P. Elustondo is with IBM Argentina, Buenos Aires, Argentina.

S. Kipnis is with IBM Israel Science and Technology, MATAMAdvanced Technology Center, Haifa 31905, Israel (e-mail:

kipnis@haifasc3.vnet.ibm.com).

IEEE Log Number 9408130 .
}

for which the interleaving of stages of local computations with stages of global communication is possible (see [27]).

This paper discusses issues related to the design and implementation of a portable and tunable Collective Communication Library (CCL). This library is intended for distributed-memory parallel computers where explicit communication among processes is achieved via message-passing. In addition to point to point communication operations, such as send and receive, users can program using CCL routines for collective operations. The set of collective communication operations in CCL consists of the following routines: bcast-one-to-all broadcast, reduce-all-to-one reduction, combine-allto-all reduction, scatter-one-to-all personalized communication, gather-all-to-one personalized communication, concat -all-to-all broadcast, index-all-to-all personalized communication, prefix-scanned reduction, shift-one-to-one cyclic permutation, and sync-barrier synchronization. A complete listing with brief descriptions of the functionality of all the CCL routines is provided in the Appendix.

The CCL was designed for the new IBM line of scalable parallel computers. (The first computer of this line of products, the IBM 9076 Scalable POWERparallel System 1 (SP1), has been announced recently.) Most of CCL is implemented as part of the parallel application programming interface of SP1.

Over the past few years, a large number of programming environments and communication libraries for parallel computers have been developed, including PVM [8], Linda [16], PICL [29], PARMACS [31], Zipcode [35], Express [37], the nCUBE/2 library [34], the CM-5 library [36], and the iPSC/860 library. The design and implementation of the CCL adopts some of the popular communication concepts that already exist in many of these libraries, and, in addition, it provides several novel aspects. In this paper, we mainly focus on three novel aspects in the design and implementation of CCL: 1) the introduction of process groups, 2) the definition of semantics that ensures correctness, and 3) the design of new and tunable algorithms based on a realistic point-to-point communication model.

For simplicity and clarity of the discussion in this paper, we only address scenarios in which dynamic process creation or deletion in run-time is not permitted. The CCL routines can either operate over the entire set of processes that are created 
at the beginning of an application or over user-specified groups of processes. For example, a user can view several processes as forming a two-dimensional array and performing independent broadcast operations in different columns of this array. Moreover, the CCL routines can also operate on groups of processes that are determined dynamically, namely, at runtime. To facilitate the use of such groups of processes, CCL includes routines that enable the definition and the handling of dynamic process groups. Section II examines issues related to process groups in CCL.

The notion of creating and using process groups has been known in the distributed computing community, such as in the V system [17], ISIS [9] and Transis [2]. In the parallel applications environment, the support for creating and using process groups for collective communication routines has been very limited. For instance, most libraries on hypercubes only support broadcasts within subcubes. Although Express also supports collective communication within an arbitrary group of processes, there is no support in creating process groups by partitioning an existing group into subgroups based on a local value supplied by each process. Such a notion and its use in parallel programming was first suggested in [3], [4], and our work influenced the introduction of this notion in an earlier MPI proposal [22] and the recent MPI proposal [21].

A major goal in the design of CCL was the creation of a truly portable library that can run correctly and efficiently on a wide range of parallel and distributed computer architectures, regardless of the topology of the interconnection network and the speeds of the processors and the communication network. Also, the CCL can be easily implemented on top of messagepassing libraries such as IBM EUI [28], IBM VIPER Operating System [25], [30], Parasoft Express [37], and PVM [8].

The CCL is a software layer that can sit on top of point-topoint communication primitives. A crucial step in the design of CCL was to define a simple and realistic model for the underlying point-to-point communication. This allows CCL to be both portable and efficient over a wide range of parallel machines. This model is discussed in Section III. In particular, Section III examines several fundamental issues related to the semantics of collective communication operations over Process Groups.

For CCL to be scalable and efficient on a variety of parallel machines, the algorithms designed for collective communication operations must be tunable and exploit the characteristics of the particular parallel computer in a way that is hidden from the user. In Section IV, we address issues related to the design of tunable algorithms for collective communication operations in CCL. Finally, in Section V we present some concluding remarks.

\section{PROCESS GROUPS IN CCL}

Process Grouping is a mechanism for grouping processes into logical sets and for manipulating and communicating among processes in such sets. This section describes the concept of Process Groups in CCL, which is based on a similar concept that was presented in [3]. (In the IBM EUI documentation [28], Process Groups are called Task Groups.)

\section{A. Defining Process Groups}

A Process Group is an ordered set of processes that has a system-wide unique name. It can be operated upon as a single object. Each Process Group is identified by a unique Process Group Identifier (PGID) in an application program. All the processes that are created at the initialization of an application belong to the predefined Process Group ALL. In addition, each process has a unique Process Identifier (PID) named mypid which could be identified with the singleton set consisting only of that process.

There are two mechanisms in CCL for defining new Process Groups. One mechanism can be used to define a new Process Group by providing an explicit list of process identifiers (PIDs) that comprise the new Process Group. This mechanism requires each member process of a to-be-formed new Process Group to know the PIDs of all the processes in the group. Using this mechanism, all the processes that comprise a new Process Group must call the group () routine:

$$
\text { pgid }=\operatorname{group}(\text { size, list, label }) \text {; }
$$

The usage of label will become clear later. The call to group () must be made by each member process of the to-beformed Process Group, and each member process must supply the same list of PIDs and in the same order. Processes that belong to the new Process Group obtain a unique PGID for this group. Processes that do not wish to be part of a new Process Group do not call the group () routine.

The other mechanism for defining new Process Groups in $\mathrm{CCL}$ is by partitioning existing Process Groups according to some criteria. This mechanism does not require the members of a to-be-formed Process Group to know the PIDs of all the processes that will comprise this group. When partitioning an existing Process Group G, several new Process Groups are created as follows. Every process in the group $G$ supplies a local integer value to myval. All the processes that supply the same value to myval are made members of the same new Process Group. The partitioning of an existing Process Group is performed by the partition() routine:

$$
\text { pgid }=\text { partition(G, myval,key); }
$$

The call to partition () must be made by every member of the existing Process Group G. No member of the existing group $G$ returns from this call until all processes in group $G$ have made the call. (In that sense, the call to partition () imposes an implicit barrier synchronization on the members of $G$ ). This call results in partitioning the members of group $G$ into as many new groups as the number of distinct integer values supplied by the member processes.

A process can participate in several group () or partition() calls and, thus, be a member of several different Process Groups. Each call to group() or partition() may correspond to creating Process Groups based on a new criterion. Each such call, therefore, creates unique PGID's for the resultant groups.

All the processes that belong to a particular Process Group $\mathrm{G}$, regardless of whether the group was created by calling group () or partition(), are ranked from 0 to $n-1$, 
where $n$ is the size of the group. The ranking of the processes in a Process Group that was created by a call to group () is the same as the order of the PIDs in the list supplied to the group () call. The ranking of the processes in a Process Group that was created by a call to partition() is determined by the additional parameter, key, that is supplied to the partition() call as an input argument. Ties in the ranking as a consequence of calling partition() are to be broken by processes' ranking in the parent group.

For example, suppose that there are 7 processes, with PID's from 0 to 6 (this is the ALL group). For convenience, we refer to a process with an even (resp. odd) PID as an even (resp. odd) process. Suppose that we want to create the following two types of groups while maintaining the original ordering: $G$ in each even process is defined as the group that consists of all even processes and $G$ in each odd process is defined as the group that consists of all odd processes. There are two ways to do this. The first is to have the following code:

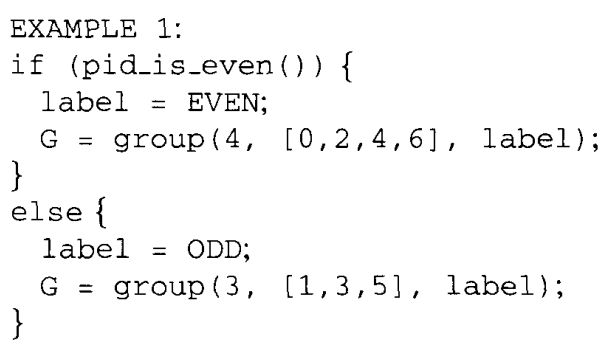

The second approach is to use partition. The code in this case is:

$$
G=\text { partition(ALL, is_mypid_odd,key); }
$$

Consider another example where all processes are partitioned into two groups depending on some locally computed value (temperature). The code is

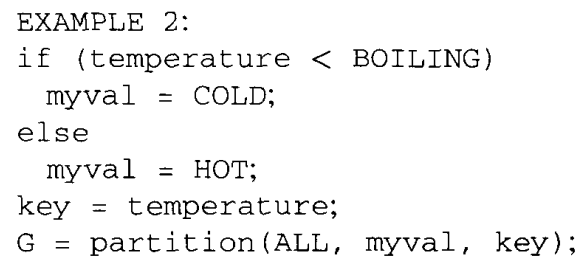

In this example, each of the two newly formed groups is sorted according to the ascending order of key, which was assigned the integer value of temperature. $\mathrm{CCL}$ also provides utility routines for processes to determine the size of a Process Group (getsize $(G)$ ), to obtain the list of PIDs in a given Process Group (getmembers $(G)$ ), to query the rank of a process in a Process Group (getrank (G, pid)), and to query the PID of a process with a given rank in a Process Group (getpid(G, rank)). Finally, the user can call get label (G) to obtain the label supplied to group () and the my-val supplied to partition().

\section{B. Using Process Groups}

Once established, Process Groups allow entire collections of processes to be identified and manipulated in a single call. Note that when different actions are required for different disjoint groups, the user can use label to distinguish groups. For example, the following code may follow the code of Example 1 above.

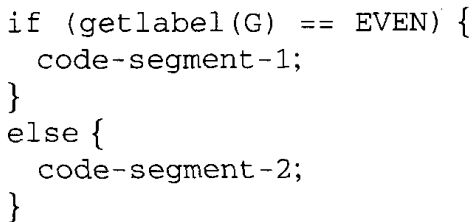

As another example, the following code may follow the code of Example 2 above.

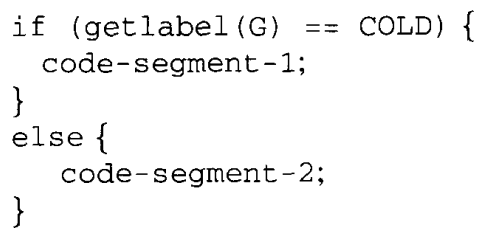

A group-wide transaction of particular interest is collective communication over all the members of a Process Group. For example, a group-wide reduction operation, which takes the PGID of a Process Group as a parameter, can be written as

$$
\text { combine(G, refunc(), data, result); }
$$

Such a routine could implement a reduction, using a supplied associative function $r$ func ( ) to combine data from the members of Process Group $\mathrm{G}$, and return the final result of the reduction to each member in the buffer result. All processes in the group make the call with the same actual parameters $G$ and $r$ func $(>)$. Similarly, a group-wide broadcast operation can be written as

$$
\text { bcast(G, orig, data); }
$$

This routine would implement a broadcast communication from a particular member of the Process Group G, whose PID is orig, to all of the other members of G. All processes in the group make the call with the same actual parameters $G$ and orig, otherwise the user's program is erroneous.

A participating process can proceed past the call to the collective communication immediately after it has finished its participation in it (even though the communication as a whole may still be in progress). For example in a grid-type problem domain, consider the sequence of calls, as shown at the top of the next page, where row_pgid and Col_pgid represent two Process Groups for each of the processes invoking the two partition() calls. Since row_pgid and COl_pgid are not disjoint, the second broadcast operation may be partially completed in parallel with the first one. The program execution order guarantees that the processes in row_pgid $\cap \mathrm{col}$ _pgid will reach the second broadcast only after they finish their participation in the first broadcast. CCL prevents possible mixing of messages between the two broadcast operations by using the semantics and properties described in Section III so that the underlying communication subsystem can distinguish between the two sets of messages. 


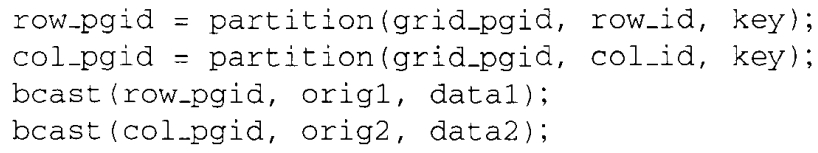

\section{Unique Process Group Identifiers}

The actual value of a PGID is determined by CCL, and user code that depends on the actual value of a PGID (such as branching based on the value of a PGID) is not allowed. That is, such code may operate correctly with one implementation of CCL and not with another. On the other hand, the user is allowed to check equality of PGID's and to include PGID's in messages sent from one process to another. As a result, CCL must guarantee that all the processes in a single Process Group use the same system-wide unique PGID, and that distinct Process Groups have distinct PGID's (whether or not they have any processes in common).

We now describe a simple mechanism for generating PGID's that are system-wide unique. Each process maintains a local counter, which is initialized to 0. Logically, a PGID consists of two fields: (counter, pid). When a new Process Group is created, the process that has rank 0 does the following three things in order: 1) determines the PGID by pairing its local counter and its PID, 2) broadcasts the value of the PGID to all the processes in the Process Group, and then 3) increments its local counter. This scheme guarantees system-wide uniqueness of all the PGID's in CCL.

The implementation of the above mechanism in CCL is slightly different. Because most existing communication subsystems have relatively large start-up costs compared to the transfer times, CCL appends the value of the local counter of each process to the control information communicated during the setup of a Process Group. This eliminates the extra communication steps needed for broadcasting the PID and the counter of the process with rank 0 , since each process has all the information needed to calculate the PGID of the new Process Group to which it belongs.

\section{Common Group Structure Routines}

Recently, a set of Common Group Structure (CGS) routines has been proposed as an extension to CCL [11]. The CGS routines make use of process group routines for defining process groups that arise in algorithms with grid and hypercube structures. Once these grid-structured and hypercube-structured groups have been defined, the standard CCL routines can be used within the structured groups to perform collective communication operations. The CGS routines also include utilities for converting between 1-dimensional and higherdimensional addresses.

As an example, the FORM2DGRID routine takes an existing group, views it as an $X \times Y 2 \mathrm{D}$-grid (where $X$ and $Y$ are specified by the user), and partitions it into a set of nonoverlapping groups corresponding to the columns of a twodimensional grid and also into a set of nonoverlapping groups corresponding to the rows of a two-dimensional grid. Thus, each process receives two new PGID's, one for the column in which it is located and one for the row in which it is located in the two-dimensional grid.

\section{Semantic Issues IN CCL}

This section discusses several issues related to the semantics of point-to-point communication and of collective communication operations on Process Groups. These issues are fundamental to the design of CCL and of similar libraries.

\section{A. Send and Receive Semantics}

In order for CCL to be portable, it is important that it is based on a simple model of point-to-point communication that is available on a wide range of machines.

The basic model of point-to-point communication consists of a single send operation and a single receive operation. It is assumed that a process can send/receive to/from any other process in the parallel machine. Specifically, for the purpose of defining send and receive semantics here and modeling the communication complexity in the next section, we treat the underlying topology as if it is fully connected. In addition it is assumed that the send and receive operations have the following five properties:

1) The operations are blocking, namely, a blocking send operation and a blocking receive operation are used. The blocking send operation is a send that does not return to the user until the message has been copied out of the user's buffer. It should be pointed out that blocking send is different from synchronous send in which the send does not return to the user until a matching receive operation has been issued. ${ }^{1}$ The blocking receive operation is a receive that does not return to the user until the requested message has arrived and has been placed in the user's buffer.

2) The communication subsystem may, but is not required to, buffer any or all of the messages. Thus, a send operation may or may not block until a matching receive is issued.

3) The receive operation is a receive-by-source, where the receiver specifies the desired source of the message. Only a message from the specified source can be delivered to the receiver. The desired source must always be specified and the use of a wildcard value for the source parameter is not allowed.

4) Point-to-point communication between any two processes is required to be FIFO such that multiple mes-

'Some researchers use the term blocking send to indicate what we have defined as synchronous send. 
sages sent from one process to another are received in the same order in which they were sent. However, the send and receive operations are asynchronous, in the sense that no bounds are assumed on the relative speeds of the processes or the time required to pass messages between processes.

5) Different send operations issued from multiple source processes to a single destination process are noninterfering. This means that the existence of messages that the receiver does not wish to select (because these messages do not match the source field specified by the receive operation) cannot prevent the reception of a message that the receiver does wish to receive. For example, if processes 1 through $n-1$ each sends a message to process 0 and if process 0 issues a receive in order to receive a message from process $n-1$, then this receive operation will get the message sent from process $n-1$ even if all of the other messages were sent earlier. While this noninterference property seems very natural, it could be violated by a system that stores messages in buffers on the receiver side, since such buffers could be filled by messages other than the one that the receiver selects to receive.

Although the basic model of point-to-point communication defined above is sufficient for implementing all of the CCL routines, the actual implementation of CCL uses two additional features that are found in many systems. These two features are a send-receive operation and the usage of message tags. ${ }^{2}$ We next discuss these two additional features and their merits in implementing CCL.

The send-receive operation simultaneously sends a message to one process and receives a message from another process without requiring additional buffer space for either of the messages. CCL uses the send-receive operation for implementing shift operations within Process Groups. The shift operation may create a cycle of processes, each of which is sending a message to its successor in the cycle and receiving a message from its predecessor in the cycle. If a send-receive operation is not used, then the send and the receive operations must be paired carefully to avoid deadlocks. (A deadlock can occur if send operations are forced to wait for matching receive operations that are never issued.) For example, to avoid deadlocks in shift operations, one can pair the send and receive operations, if the cycle is of even length, as follows. Processes in even positions in the cycle would perform a send followed by a receive, while processes in odd positions in the cycle would perform a receive followed by a send. (Cycles of odd length can be handled with one more round of communication.) Although such an algorithm is possible in CCL, since each process knows its position in the cycle, it is much simpler and more efficient to use a send-receive operation for implementing the shift.

Some point-to-point communication libraries allow the use of wildcard values in the source parameter of a receive call. Even if wildcard values are not used in the implementation of

\footnotetext{
${ }^{2}$ Some researchers use the term type instead of tag.
}

CCL, there is still the risk that a message sent as part of the implementation of a CCL call will be mistakenly received by a receive call with a wildcard source issued by the user. An additional mechanism is needed to distinguish CCL messages from user messages. Message tags can provide such mechanism. These are values that are appended to the content of point-to-point messages. Tags are typically used to distinguish between different types of messages in an application, as well as between multiple messages sent to a destination from the same source. Each send operation specifies a value in the tag field of the message, and each receive operation specifies a desired tag value to be matched with the received message.

We assume that a mechanism is available to allocate tag ranges to various libraries, so that user messages and messages generated by $\mathrm{CCL}$ have disjoint tag ranges. If wildcard tag values are allowed in receive operations, one also needs to make sure that user receive operations cannot match tag values used by CCL. This can be achieved either with an include/exclude mechanism for wildcard tag ranges, as provided by Express [37] or with an additional context mechanism, as provided by Zipcode [35].

\section{B. Implementation Correctness}

There are many issues related to a correct implementation of CCL. These issues include, for example, separating user point-to-point messages from CCL point-to-point messages, guaranteeing that the correct messages be delivered for collective communication operations in overlapping Process Groups, and distinguishing between messages that belong to different collective communication operations in the same group.

We will concentrate on the correct implementation of the CCL routines assuming the basic model of point-to-point communication satisfying the five properties listed above. The key to this implementation is the notion of deterministic code. Code will be said to be deterministic if $i$ ) it uses only the basic send and receive primitives given above, $i i$ ) it does not deadlock even when every send operation is forced to wait for a matching receive operation, and iii) all local computations are deterministic. It has been proven that a deterministic program will always behave identically, regardless of the system on which it is implemented [19]. The correctness of the CCL implementation follows from the fact that each CCL routine is deterministic. As a result, each CCL routine is guaranteed to operate correctly when it is run in isolation.

Of course the CCL routines must also operate correctly when they are run as part of an application consisting of point-to-point communication and multiple CCL operations. The noninterference property (property 5 above) guarantees that the presence of application-level point-to-point messages and other CCL operations does not prevent the successful completion of a CCL routine.

Furthermore, the semantics of CCL assume that each CCL routine may imply, but need not imply, a barrier synchronization of the processes calling the CCL routine (this issue is explored in more detail in the following subsection). Therefore, one requirement of a correct program using CCL is that 
any CCL operation that involves processes $p$ and $q$ should be called in the same order by processes $p$ and $q$, and no pointto-point communication between processes $p$ and $q$ should overlap a CCL operation involving $p$ and $q$. As a result, it follows from the FIFO property of the communication (property 4 above) that in a correct application, messages from CCL operations on overlapping groups, repeated CCL operations on the same group, and application-level point-topoint communication cannot be erroneously received by $\mathrm{CCL}$, and vice-versa.

\section{Barrier Semantics}

As discussed above, for program correctness, each CCL operation should be viewed as possibly imposing a barrier synchronization on its participants. However, from the performance point of view, imposing a barrier synchronization on all the participants in CCL operations may be undesirable. To address this problem, CCL allows two modes of operation: barrier mode and nonbarrier mode. Selection between these two modes is done globally at the beginning of an application. The default mode is the barrier mode. When a CCL operation is executed in barrier mode, no process can complete its call to the routine until all the processes in the relevant Process Group have executed their (corresponding) calls to the same routine. Therefore, when an operation is executed in barrier mode, there must exist some point in time at which all the processes in the group are simultaneously executing the same operation. In contrast, when an operation is executed in nonbarrier mode, each process blocks only until it has completed its participation in the operation. Notice that the role of each process may depend not only on the semantics of the operation but also on the specific algorithm used to implement it.

The differences between the two modes may imply differences in the behavior of an application program. In a nonbarrier mode, processes may return more quickly from calls to CCL routines, resulting in a better performance. However, there is also some danger in using nonbarrier mode routines as in some CCL routines the role of each process depends also on the particular algorithm used to implement the routine. As long as the user's program is correct and deterministic, then nonbarrier mode routines will operate correctly (as argued above) and in an implementation-independent manner. However, if wildcard point-to-point communication is used, then the behavior of a nonbarrier mode routine may be implementation-dependent. As an example, consider the following piece of code, in which $G$ is the group identifier of a Process Group consisting of processes $a, b$, and $c$. In this example, each brecv is a blocking receive operation with a wildcard source parameter.

$\begin{array}{ccc}\text { process a } & \text { process b } & \text { process C } \\ \text { bsend(c) } & & - \\ \operatorname{bcast}(G) & b \operatorname{brecv}(*) \\ & \text { bsend(c) } & \operatorname{bcast}(G) \\ & \operatorname{brecv}(*)\end{array}$

Assume that the source of the bcast operation is process b. If barrier semantics are used for the broadcast operation, then the first receive of process $c$ will receive the message sent by process $a$, and the second receive of process $c$ will receive the message send by process $b$. On the other hand, if process $b$ can exit the broadcast call before process $c$ starts executing the broadcast, then it is possible for the messages to be received in the reverse order: process $c$ receives before the broadcast the message that $\mathrm{b}$ sent after the broadcast, and receives after the broadcast the message that a sent before the broadcast!

In summary, nonbarrier mode CCL routines may exhibit implementation dependent blocking behavior when nondeterministic point-to-point communication operations are allowed. Furthermore, even if no wildcard point-to-point communication is performed, a program that would deadlock with barrier mode CCL routines may complete successfully with nonbarrier model CCL routines. Therefore, barrier mode should be used when debugging code (that is, in identifying bugs that may not be caught in nonbarrier mode). Once a program is known to operate correctly in barrier mode, it is possible to switch to nonbarrier mode for better performance (provided that the barrier mode is not required for the correctness of the program). Finally, notice that it is always possible to use an explicit synchronization (i.e., to call the routine sync) when a barrier is needed.

In our CCL, some collective communication operations have semantics that imply a barrier, while others do not. Operations that imply a barrier are combine, concat, index, and sync. Thus, the implementation of these operations is the same for barrier and for nonbarrier modes. Other operations that do not imply a barrier are bcast, reduce, scatter, gather, prefix, and shift. In nonbarrier mode, the latter operations (except shift) are implemented with a fan-in tree to a particular destination or a fan-out tree from a particular source. For implementing these operations in barrier mode, we use two internal partial synchronization routines: syncin (dest, G) which builds a fan-in tree in the group $G$ that converges at process dest, and sync-out (srC, G) which builds a fan-out tree in the group $\mathrm{G}$ that diverges from process src. A barrier mode for the latter operations is implemented by performing a sync-in () before a fan-out type operation (like bcast and scatter), or by performing a syncout () after a fan-in type operation (like reduce, gather, and prefix). Notice that for the prefix operation, the fanout tree is rooted at the highest ranked process in the group. Finally, regarding the Process Group creation operations, partition() implies a barrier mode within the parent group while group() does not.

\section{Develop and Run Modes}

In addition to the choice between barrier and nonbarrier modes, the user can select between develop and run modes. In develop mode, CCL performs more detailed consistency checkings of the parameters that the user specifies for CCL calls. This increases the overhead of the CCL operations and may require more communication between processes. In run mode, many of the consistency checkings are suppressed, and only checkings that are required by the semantics of the operations are performed. The default in CCL is run mode and 
the mode can be changed by the user. Examples of consistency checkings that are done only in develop mode are checking the correctness of the source, the destination and the message length supplied to a CCL routine, as well as checking the membership list of a Process Group to be valid.

\section{PERFormance IsSUES IN CCL}

This section discusses several performance issues and describes some of the algorithms used in CCL. The design of communication algorithms for CCL attempts to address both the efficiency and the portability of the communication operations. In the discussion of the communication algorithms in this section, we assume one process per processor.

\section{A. Communication Model}

In designing algorithms for the CCL operations, we assume a model of a fully-connected message-passing system in which each process can communicate directly with any other process and every pair of processes are equally distant. We also assume that each process can send one message and, simultaneously, receive another message in the same communication step. (This is usually done using a send-receive operation found in many parallel systems.) In most existing messagepassing parallel systems, the time for sending an $m$-byte message from process $p$ to process $q$, without congestion, can be modeled as $T=t_{s}+m t_{c}$, where $t_{s}$ is the overhead (startup time) associated with each send and/or receive operation, and $t_{c}$ is the communication time for sending each additional byte (or any appropriate data unit).

Such a fully-connected model addresses emerging trends in many modern distributed-memory parallel computers and message-passing communication environments. These trends are evident in systems such as Thinking Machines' CM-5 [36], Intel's Paragon [38], NCUBE's nCUBE/2 [34], MIT's J-Machine [20], IBM's Vulcan [10], [39], and the recently announced IBM's Scalable POWERparallel System 1 (SP1), and in environments such as Express [37], PARMACS [31], PICL [29], Zipcode [35] and Venus [4]. These systems and environments generally ignore the specific structure and topology of the communication network and assume a fully-connected collection of processes, in which each process can communicate directly with any other process by sending and receiving messages. The fact that the model does not assume any single topology makes it more general and flexible. This model, for instance, allows the creation of algorithms that are portable between different machines, that can operate within arbitrary and dynamic subsets of processes, and that can operate in the presence of faults (assuming connectivity is maintained). In addition, algorithms developed for this model can also be helpful in designing algorithms for specific topologies.

To examine the performance of communication algorithms, we define the following three communication complexity measures:

- $C_{1}$ : the number of communication steps required by an algorithm. $C_{1}$ is an important measure when the communication start-up time is high relative to the transfer time of one unit of data and the message size per send/receive operation is relatively small.

- $C_{2}$ : the amount of data (in the appropriate unit of communication: bytes, flits, or packets) transferred in sequence by any process. $C_{2}$ is an important measure when the start-up time is small compared to the message size.

- $C_{3}$ : the total amount of data communicated over the network (in the appropriate unit of communication: bytes, flits, or packets). Measures $C_{1}$ and $C_{2}$ do not address the issue of load on the network. $C_{3}$ also considers the fact that communicating more data over the network causes the network to become more congested.

Thus, under the fully-connected model, an algorithm has an estimated communication time complexity of $T=C_{1} t_{s}+$ $C_{2} t_{c}$. The term $C_{3}$ does not affect the complexity here, but may be helpful in estimating the congestion behavior of a real parallel machine. For instance, one can model $T=C_{1} t_{s}+C_{2} t_{c}+f\left(C_{3}\right)$, where the function $f$ can be derived for a given machine either explicitly or by curve-fitting experimental results.

It should be noted that there are more detailed communication models, such as the Postal model [5] and the LogP model [18], which further take into account that a receiving process generally completes its receive operation later than the corresponding sending process finishes its send operation. However, designing efficient algorithms for these models seems to be more complicated. Another important issue is the uniformity of the implementation. For example, in the $\log P$ model, the collective communication algorithms are designed based on $P$ (the number of processors). The optimal algorithms for two distinct values of $P$ are sometimes very different. This presents a challenge if the goal is to support collective communication algorithms for various sizes of process groups using the same collective communication library.

\section{B. Tunable Algorithms}

One goal in the design of the algorithms for CCL was that they be tunable, that is, that they exhibit a trade-off between the different communication complexity measures. This goal is important for such a library to be both portable and efficient. In the following discussion, we use $n$ to denote the number of processes (processors) involved in a CCL operation, and we use $m$ to denote the size of data each process has initially. All CCL routines can be implemented with $C_{1}=\left\lceil\log _{2} n\right\rceil$ communication steps, which is optimal for this measure. (The shift routine can be implemented optimally in one communication step, by using the sendreceive operation.) However, when designing an algorithm to minimize $C_{1}$, some of the corresponding terms for $C_{2}$ or $C_{3}$ may not be optimal. In general, there are tradeoffs between $C_{1}$ and $C_{2}$, or between $C_{1}$ and $C_{3}$.

As an example, consider the index operation. A straightforward implementation of the index operation can be achieved with $C_{1}=n-1$ communication steps and $C_{2}=$ $m(n-1) / n$ units of data. This is done simply by sending the data directly from source to destination. Namely, each process sends $m / n$ units of data to $n-1$ other processes. Another 
approach is by using a different radix for representing the PIDs of the processes (the straightforward approach is using a radix $n$ representation). In the case of a radix 2 representation we get $C_{1}=\lceil\log n\rceil$ communication steps and $C_{2} \approx(m \log n) / 2$ units of data. In general, by choosing an appropriate radix $r$, the index operation can be implemented with $C_{1} \approx$ $(r-1) \log _{r} n$ communication steps and with $C_{2} \approx(m(r-$ 1) $\left.\log _{r} n\right) / r$ units of data. Hence, it is possible to implement a parameterized algorithm which can be tuned according to the start-up time $t_{s}$, per-byte transfer time $t_{c}$, the message size $m$, and possibly the number of "parallel ports" that can support concurrent sends and receives effectively (see [15]).

As a second example, consider the broadcasting problem. The algorithm for the bcast routine is straightforward when the size of the data is $m=1$, that is, when the source of the broadcast has one item to broadcast. In this case, a divide-and-conquer algorithm provides an optimal solution, also known as recursive doubling. However, the broadcasting problem becomes much more complicated when there are $m>1$ units of data to broadcast. In this case, a lower bound on the time is $\lceil\log n\rceil t_{s}+(m+\lceil\log n\rceil-1) t_{c}$. The common divide-and-conquer algorithm based on a binomial tree takes $\lceil\log n\rceil\left(t_{s}+m t_{c}\right)$ time, which may be far from optimal. When $n$ is a power of two, an algorithm based on $\log n$ edge-disjoint spanning trees on a $(\log n)$-cube is given in [33], [32]. This algorithm requires $C_{2}=m+\log n-1$, which is optimal, and takes $T=\left(\sqrt{m t_{c}}+\sqrt{\log n t_{s}}\right)^{2}$ time. For arbitrary values of $n$, an algorithm based on the generalized Fibonacci trees was given in [13] with $C_{2} \leq m+\log n+3 \log \log n+15$. More recently, an algorithm based on edge-disjoint spanning trees of cascaded decreasing-size hypercubes was given [6] with nearly optimal $C_{2}=m+\lceil\log n\rceil$. The reduce operation is usually implemented in a similar manner to the bcast operation by reversing the flow of the messages.

As another example, consider the combine and prefix operations. These operations exhibit an interesting trade-off between measures $C_{1}$ and $C_{3}$. On one hand, these operations can be implemented with $C_{1}=2\lceil\log n\rceil$ and $C_{3}=O(m n)$ using a reduction tree followed by a broadcast tree. On the other hand, these operations can be implemented with $C_{1}=\lceil\log n\rceil$ and $C_{3}=O(m n \log n)$ using a butterflytype or circulant-graph-type communication pattern. In fact, hybrid algorithms for these operations exist. For instance, a hybrid algorithm for the combine operation requires $C_{1}=$ $\lceil\log n\rceil+k$ communication steps and communicates $C_{3}=$ $m\left(n / 2^{k}\right)\left(\log n+2^{k+1}-k-2\right)$ units of data (see [1]).

The scatter and gather operations resemble the bcast and reduce operations in their functionality. However, in terms of their performance, these operations can be implemented with $C_{1}=\lceil\log n\rceil$ communication steps and $C_{3} \approx(m \log n) / 2$ units of data, or, alternatively, they can be implemented with $C_{1}=n-1$ communication steps and $C_{3}=m(n-1) / n$ units of data.

\section{An Optimal Algorithm for Concatenation}

In this subsection, we outline an algorithm for the concat operation. (In [14] we showed that this algorithm is optimal with respect to measures $C_{1}, C_{2}$ and $C_{3}$.) In the concat (all-

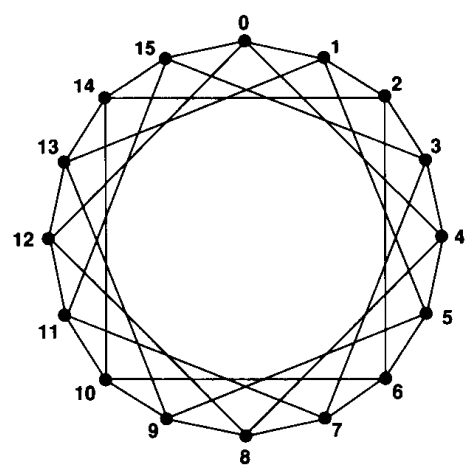

Fig. 1. A circulant graph with 16 nodes and offsets 1 and 4 .

to-all broadcast) operation among $n$ processes, each process has a fixed-size message (also called a data block) that it needs to broadcast to the other $n-1$ processes. Thus, at the end, each process has all $n$ data blocks. Because $n$ need not necessarily be a power of two, the simple optimal algorithm for concatenation, which is based on a butterfly-type communication pattern, cannot be directly used.

The optimal algorithm for the concat operation is based on the structure of a circulant graph. A circulant graph $G(n, S)$ is characterized by two parameters: the number of nodes $n$, and a set of integer offsets $S$. The nodes of the graph are labeled from 0 through $n-1$. Each node $i$ is connected to nodes of the form $((i \pm s) \bmod n)$ for all $s \in S$ (see [24]). Circulant graphs are an important class of networks which can be used as fault-tolerant networks for many other networks [12], [23]. We use a circulant graph with power-of-two offsets $S=\left\{1,2,4, \cdots, 2^{k-1}\right\}$, where $k=\lceil\log n\rceil$, as a structure for the concat algorithm [14] (see Fig. 1).

The algorithm consists of $k$ steps. In step 0 , each process $i$ sends its data block to process $(i-1) \bmod n$, receives a data block from process $(i+1) \bmod n$, and appends the received data block to its current data. In general, in step $j$, for $0 \leq j \leq k-2$, each process $i$ sends all its current cumulated $2^{j}$ data blocks to process $\left(i-2^{j}\right) \bmod n$, receives $2^{j}$ data blocks from process $\left(i+2^{j}\right) \bmod n$, and appends the received data to its cumulated data blocks. In the last step, step $k-1$, each process $i$ sends only the first $n-2^{k-1}$ blocks of data that it cumulated to process $\left(i-2^{k-1}\right) \bmod n$, and it receives the same number of data blocks from process $\left(i+2^{k-1}\right) \bmod n$. Fig. 2 presents an example of this algorithm.

In general, all the homogeneous operations (operations for which there is no notion of a distinct source and/or destination, [26]) in CCL, can be implemented with the minimal number of start-ups ( $\lceil\log n\rceil$ communication steps) using the same circulant graph structure. Examples of other such operations are combine, index, and sync. An algorithm for the combine operation with $T=\lceil\log n\rceil\left(t_{s}+m t_{c}\right)$, which is optimal with respect to $C_{1}$ (and also to $C_{2}$ when $m=1$ ), appears in [7].

\section{Specialized Algorithms}

For certain CCL operations, the best-known algorithms for the case when $n$ is a power of 2 may perform better than 


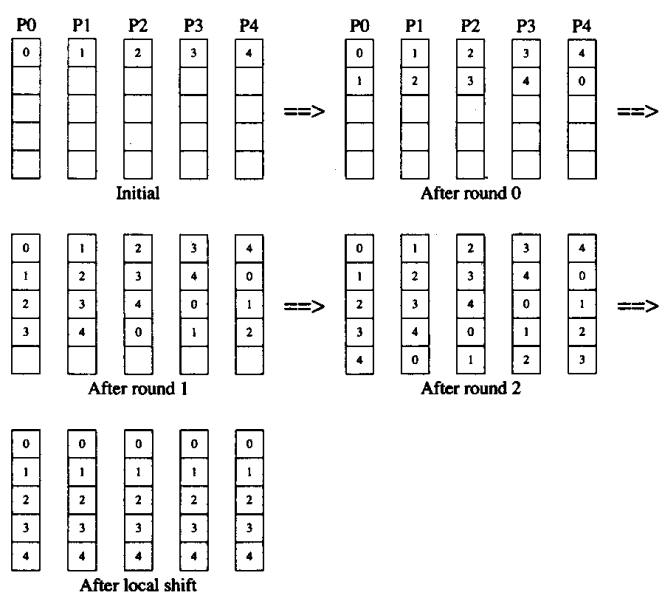

Fig. 2. An example of the concatenation algorithm with 5 processors.

algorithms for the general case. Since, in many situations, $n$ is a power of 2 , it is worthwhile to implement this case separately. For instance, the concat algorithm for $n$ that is a power of two can use a well-known hypercube recursive exchange algorithm which eliminates shifting local arrays at the end of the operation. As another example, when $n$ is a power of two, the bcast algorithm described in [32] is substantially simpler, in terms of local data structures and control, than the algorithm for arbitrary values of $n$ described in [6].

\section{CONCLUSIONS}

We have described the main issues that we have encountered in designing and implementing a Collective Communication Library (CCL) for the recently announced IBM Scalable POWERparallel System 1, (SP1). We have focused on three novel aspects in the design and implementation of CCL: the introduction of process groups, definition of semantics that ensures correctness and the design of novel algorithms based on a realistic point-to-point communication model. Each of these novel aspects suggests interesting avenues for further learning and research.

\section{APPENDIX}

\section{LIST OF CCL ROUTINES}

CCL consists of two parts, the collective communication routines (CC part) and the process group routines (PG part which is called TG for task group in the EUI document.) The $\mathrm{CC}$ routines provide the functionality of operations involving collective communication, and the PG routines provide the functionality of specifying and manipulating groups.

The available $\mathrm{CC}$ routines are:

bcast (): Single source broadcast, i.e., broadcast a message from one process to all tasks in a group.

reduce (): Apply an associative (but not necessarily commutative) reduction operation on all the processes in a group, and place the reduction result in one user-specified process. combine (): Apply an associative (but not necessarily commutative) reduction operation on all the processes in a group, and place the reduction result in each of the processes in the group.

scatter(): Distribute distinct messages from a single source to each process in a group.

gather (): Gather distinct messages from each process in a group to a single destination process.

concat (): Concatenate to all processes in a group. Each process in a group performs a bcast within the group.

index(): Each task in a group performs a scatter operation.

prefix (): Parallel prefix operation. It is sometimes called scan.

shift (): Shift data up or down some number of steps in a group.

sync (): Barrier synchronization in a group.

The PG routines are summarized below.

group (): Create a process group by explicitly specifying the processes in the group.

partition(): Define a process group by partitioning an existing group based on a locally supplied integer label.

getsize (): Get the size (the number of processes) of an existing process group.

getmembers (): Get the ordered array of process ids of an existing process group.

getrank (): Get the rank of a process in a process group. getpid(): Get the process id of the process with a certain rank in a process group.

get label (): Get the label (which was supplied by a user when the group was formed) of an existing process group.

\section{ACKNOWLEDGMENT}

The authors thank the following people: D. Frye for his valuable comments and help in revising the $\mathrm{CCL}$ semantics as part of the spec in [28], M. Konstantinidou for her contributions to the initial stage of the CCL design, E. Leu for his comments on a draft of this paper, many internal and external reviewers of [28] for their valuable comments to the CCL semantics, and D. Petkovic for his constant support and help.

\section{REFERENCES}

[1] A. Aggarwal and S. Kipnis, "Message-time tradeoff for combining data in message-passing systems," IBM Res. Rep. RC-18349, Sept. 1992.

[2] Y. Amir, D. Dolev, S. Kramer, and D. Malki, "Transis: A communication sub-system for high availability," in Proc. 22nd Ann Int Symp Fault-Tolerant Computing, July 1992, pp. 76-84.

[3] V. Bala and S. Kipnis, "Efficient collective communication over dynamically determined sets of processors in massively parallel computers," IBM Res. Rep. RC-17771, Mar. 1992.

[4] _ "Process groups: A mechanism for the coordination of and communication among processes in the Venus collective communication library," in Proc. 7th Int Parallel Processing Symp, Apr. 1993.

[5] A. Bar-Noy and S. Kipnis, "Designing broadcasting algorithms in the postal model for message-passing systems," in Proc. 4th Ann ACM Symp Parallel Algorithms, Architectures, June 1992, pp. 11-22.

[6] __ "Broadcasting multiple messages in simultaneous send/receive systems," in Proc. 5th IEEE Symp Parallel, Distributed Processing, Dec. 1993. 
[7] A. Bar-Noy, S. Kipnis, and B. Schieber, "An optimal algorithm for computing census functions in message-passing systems," Parallel Processing Lett., vol. 3, no. 1, Mar. 1993.

[8] A. Beguelin, et al., "A user's guide to PVM Parallel Virtual Machine," ORNL Tech. Rep. ORNL/TM-11826, May 1992.

[9] K. Birman et al., "The ISIS system manual," Department of Computer Science, Cornell University, Ithaca, NY, Sept. 1990.

[10] J. Bruck et al., "Survey of routing issues for the Vulcan parallel computer," IBM Res. Rep. RJ-8839, June 1992.

[11] J. Bruck et al., "A proposal for common group structures in a collective communication library," IBM Res. Rep. RJ-9421, Mar. 1993.

[12] J. Bruck, R. Cypher, and C. T. Ho, "Efficient fault-tolerant mesh and hypercubes architectures," in Proc. 1992 Int. Symp. Fault-Tolerant Computing, pp. 162-169.

[13] J. Bruck, R. Cypher, and C. T. Ho, "Multiple message broadcasting with generalized Fibonacci trees," in Proc. 4th IEEE Symp. Parallel, Distributed Processing, Dec. 1992, pp. 424-431.

[14] J. Bruck, C. T. Ho, and S. Kipnis, "Concatenating data optimally in message-passing systems," IBM Res. Rep. RJ-9191, Jan. 1993.

[15] J. Bruck, R. Cypher, C. T. Ho, and S. Kipnis, "Efficient algorithms for the index operation in message-passing systems," IBM Res. Rep. RJ-9300, Apr. 1993.

[16] N. Carriero and D. Gelernter, "Linda in context," Commun. ACM, vol. 32, no. 4, pp. 444-458, Apr. 1989.

[17] D. R. Cheriton and W. Zwaenpoel, "Distributed process groups in the V kernel," ACM Trans. Comput. Syst., vol. 2, no. 3, pp. 77-107, May 1985

[18] D. Culler et al., "LogP: Towards a realistic model of parallel computation," in Proc. 4th SIGPLAN Symp. Principles, Practices of Parallel Programming, ACM, May 1993.

[19] R. Cypher and E. Leu, "Message-passing semantics and portable parallel programs," IBM Res. Rep. RJ-9654, Jan. 1994.

[20] W. J. Dally et al., "The J-machine: A fine-grain concurrent computer," in Proc. Inform. Processing 89, 1989, pp. 1147-1153.

[21] Document for a Standard Message-Passing Interface, Message Passing Interface Forum, Tech. Rep. CS-93-214, Univ. Tennessee, Nov. 1993.

[22] J. Dongarra, R. Hempel, A. Hey, and D. Walker, "A proposal for a userlevel, message-passing interface in a distributed memory environment," ORNL Tech. Rep. ORNL/TM-12231, Oct. 1992.

[23] S. Dutt and J. P. Hayes, "Designing fault-tolerant systems using automorphisms," J. Parallel. Distrib. Computing, vol. 12, pp. 249-268, 1991.

[24] B. Elspas and J. Turner, "Graphs with circulant adjacency matrices," $J$. Combinatorial Theory, no, 9, pp. 297-307, 1970.

[25] B. G. Fitch and M. E. Giampapa, "The Vulcan operation environment: A brief overview and status report," in Proc. 5th Workshop Use Parallel Processors Meteorology, ECMWF, Nov. 1992.

[26] G. Fox and W. Furmanski, "Optimal communication algorithms for regular decompositions on the hypercube," in Proc. 3rd Conf. Hypercube Concurrent Comput. Applicat. ACM, pp. 648-713, 1988

[27] G. Fox et al., Solving Problems on Concurrent Processors, Volume I: General Techniques and Regular Problems. Englewood Cliffs, NJ: Prentice-Hall, 1988.

[28] D. Frye et al., "An external user interface for scalable parallel systems: FORTRAN interface," Tech. Rep., IBM Highly Parallel Supercomputing Systems Laboratory, Nov. 1992.

[29] G. A. Geist, M. T. Heath, B. W. Peyton, and P. H. Worley, "A user's guide to PICL: A portable instrumented communication library," ORNL Tech. Rep. ORNL/TM-11616, Oct. 1990.

[30] M. E. Giampapa, B. G. Fitch, G. R. Irwin, and D. G. Shea, "Vulcan operating environment: Programmer's reference," Intern. Memorandum, IBM T. J. Watson Research Center, Yorktown Heights, NY, Mar. 1991

[31] R. Hempel, "The ANL/GMD macros (PARMACS) in FORTRAN for portable parallel programming using the message passing programming model, user's guide and reference manual," Tech. Memorandum, Gesellschaft für Mathematik und Datenverabeitung mbH, West Germany.

[32] C. T. Ho, "Optimal broadcasting on SIMD hypercubes without indirect addressing capability," J. Parallel, Distrib. Computing, vol. 13, no. 2, pp. 246-255, Oct. 1991

[33] S. L. Johnsson and C. T. Ho, "Spanning graphs for optimum broadcasting and personalized communication in hypercubes," IEEE Trans. Comput., vol. 38, no. 9, pp. 1249-1268, Sept. 1989.

[34] J. F. Palmer, "The NCUBE family of parallel supercomputers," in Proc. Int. Conf. Comput. Design, IEEE, 1986.

[35] A. Skjellum and A. P. Leung, "Zipcode: A portable multicomputer communication library atop the Reactive Kernel," in Proc. 5th Distrib. Memory Computing Conf., IEEE, Apr. 1990, pp. 328-337.

[36] Connection Machine CM-5 Tech. Summary, Thinking Machines Corporation, 1991.
[37] Express 3.0 Introductory Guide, Parasoft Corporation, 1990

138] Paragon XP/S Overview, Intel Corporation, 1991.

[39] "Vulcan system summary," Int. Memorandum, IBM T. J. Watson Research Center, Yorktown Heights, NY.

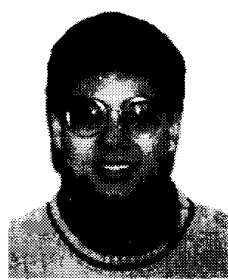

Vasanth Bala received the M.S. and Ph.D. degrees in computer science from Rice University, Houston, TX, in 1989.

Since then he has been a Research Fellow in the Concurrent Computation Lab at Caltech, Pasadena, $\mathrm{CA}$, and as a Research Staff Member at the IBM T. J. Watson Research Center in Yorktown Heights, NY. In April 1993 he joined Kendall Square Research Corporation, Waltham, MA, where he is currently a Member of the Technical Staff. His research interests include compiler optimizations for scalable parallel computers and superscalar RISC processors.

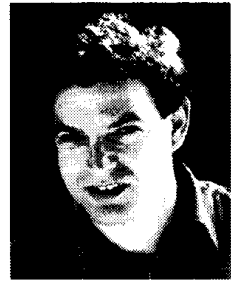

Jehoshua Bruck (S'86-M'89-SM'93) received the $\mathrm{B} . \mathrm{Sc}$ and $\mathrm{MSc}$ degrees in electrical engineering from the Technion, Israel Institute of Technology, in 1982 and 1985, respectively, and the Ph.D. degree in electrical engineering from Stanford University, Stanford, CA, in 1989.

$\mathrm{He}$ is an Associate Professor of Computation and Neural Systems and Electrical Engineering at the California Institute of Technology, Pasadena. His research interests include parallel and distributed computing, fault-tolerant computing, error-correcting codes, computation theory, and neural systems. He has extensive industrial experience, including serving as Manager of the Foundations of Massively Parallel Computing Group at the IBM Almaden Research Center, San Jose, CA, from 1990 to 1994, a Research Staff Member with the IBM Almaden Research Center from 1989 to 1990, and a Researcher with the IBM Haifa Science Center, Haifa, Israel, from 1982 to 1985.

Dr. Bruck is the recipient of a 1994 National Science Foundation Young Investigator Award, a 1992 IBM Outstanding Innovation Award for his work on "Harmonic Analysis of Neural Networks," and a 1994 IBM Outstanding Technical Achievement Award for his contributions to the design and implementation of the SP-1, the first IBM scalable parallel computer. He has received five IBM Plateau Invention Achievement Awards and holds 15 patents.

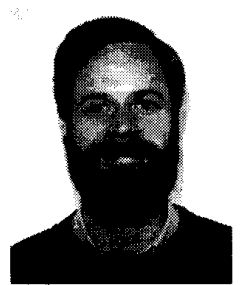

Robert Cypher (M'93) received the B.S. degree in mathematical sciences from Stanford University, Stanford, CA, in 1982, and the M.S. and Ph.D. degrees in computer science from the University of Washington, in 1987 and 1989, respectively.

He was a Research Staff Member at the IBM Almaden Research Center, San Jose, CA, and a Consulting Assistant Professor with the Stanford University Computer Science Department from 1989 to 1993. He was then a Research Staff Member with the IBM T. J. Watson Research Center, Yorktown Heights, NY, from 1993 to 1994. Currently, he is an Assistant Professor in the Johns Hopkins University Computer Science Department, Baltimore, MD. His research interests include parallel algorithms and architectures, communication protocols, the semantics of parallel programs, fault-tolerance, and the design of interconnection networks. 


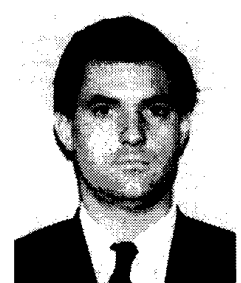

Pablo M. Elustondo received the B.S. degree in computer science from the Escuela Latino Americana de Informatica, Argentina, in 1988.

From 1989 to 1990 he was a Research Assistant at the Catholic University of Rio de Janeiro in Brazil, where he focused on the formal description of program and development concepts. In 1991 he joined the Advanced Solutions and Innovative Technology Group at IBM Argentina, Buenos Aires, where he is currently a Member of the Technical Staff. He was with the Almaden Research CenterSan Jose, CA during 1992, in the Foundations of Massively Parallel Computing Group. His primary interests were parallel languages and distributed programming tools. His current research interests include most aspects of the software development process with emphasis on object-oriented software-support tools and languages.

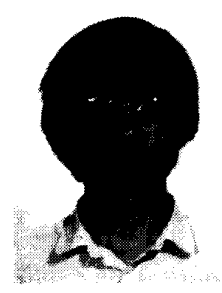

Alex Ho received the M.S. degree in civil and environmental engineering from Cornell University, Ithaca, NY, in 1983.

$\mathrm{He}$ is with the IBM Almaden Research Center, San Jose, CA. His research interests include parallel and distributed computing environments, and scientific applications in natural computation and environmental fluid mechanics.

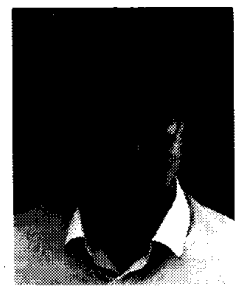

Ching-Tien Ho (S'85-M'89) received the B.S. degree in electrical engineering from National Taiwan University in 1979 and the M.S., M.Phil., and Ph.D. degrees in computer science from Yale University, New Haven, CT, in 1985, 1986, and 1990, respectively.

He joined the IBM Almaden Research Center as a Research Staff Member in 1989. He became Project Leader in 1992 and Manager of the Foundations of Massively Parallel Computing group in 1994, where he leads the development of collective communication, as part of IBM EUI and MPI, for IBM SP-1 and SP-2 paralle systems. His primary research interests include communication issues for interconnection networks, algorithms for collective communications, graph embeddings, fault tolerance, and parallel algorithms ane archtectures.

Dr. Ho was a co-recipient of the 1986 "Outstanding Paper Award" of the International Conference on Parallel Processing. He has received an IBM Outstanding Innovation Award, an IBM Outstanding Technical Achievement Award, and three IBM Plateau Invention Achievement Awards. He has six patents granted or pending. He has served on Program Committees of the IEEE Symposium on Parallel and Distributed Processing and the International Conference on Parallel and Distributed Systems. He is a member of ACM.

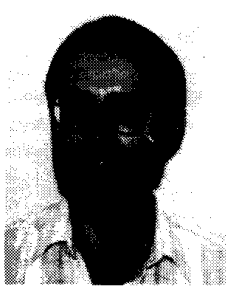

Shlomo Kipnis (M'87) received the B.Sc. degree in mathematics and physics in 1983 and the M.Sc degree in computer science in 1985, both from the Hebrew University of Jerusalem, Jerusalem, Israel. He received the $\mathrm{Ph} . \mathrm{D}$. degree in electrical engineering and computer science in 1990 from the Massachusetts Institute of Technology, Cambridge.

From 1990 to 1993 , he was a Research Staff Member at the IBM T. J. Watson Research Center, Yorktown Heights, NY. Currently, he is a Research Staff Member at the IBM Science and Technology Center, Haifa, Israel. His research interests include parallel and distributed processing, efficient computation and communication structures, VLSI, and algorithms. He has published in numerous journals and presented his work at many conferences and workshops. He is also an inventor and coinventer of two U.S. patents.

Dr. Kipnis is a member of the ACM and the IEEE Computer Society.

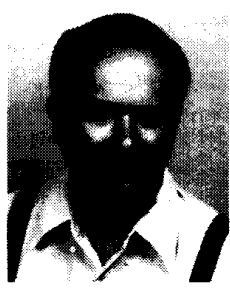

Marc Snir (SM'89) received the Ph.D. in mathematics from the Hebrew University of Jerusalem in 1979.

He is Senior Manager at the IBM T.J. Watson Research Center, Yorktown Heights, NY, where he leads the research on scalable parallel software. He was with New York University, New York, NY, on the NYU Ultracomputer project in 1980-1982 and was with the Hebrew University of Jerusalem in 1982-1986. He was recently involved in the definition of High Performance Fortran and is now involved in the definition of the Message Passing Interface. He has published on computational complexity, parallel algorithms, parallel architectures, interconnection networks, and parallel programming environments.

Dr. Snir is a member of the IBM Academy of Technology, ACM, and SIAM. 